\author{
Michał Szucki*, Józef S. Suchy***, Paweł Żak*, \\ Janusz Lelito**, Beata Gracz*
}

\title{
EXTENDED FREE SURFACE FLOW MODEL BASED ON THE LATTICE BOLTZMANN APPROACH
}

\section{INTRODUCTION}

From the CFD (Computational Fluid Dynamics) point of view, the issue of casting mould filling with liquid metal, can be treated in two ways. On one hand, in the case of simple gravity castings made in sand molds with good permeability, the effect of gas located inside a mould on the movement of liquid alloy is negligible. In that case free surface flow models (where the gas phase is not reflected) seem to be the best choice. On the other hand, in technologies such as die casting, or in the case of flows in complex gating systems, omitting the calculation of the gas phase movement can lead to significant errors. In this situation numerical models for two-phase (or multiphase) flows perform better. For obvious reasons this second approach is more universal and allows to solve more complex problems. However, in many cases these benefits are strictly connected with significant complications during the implementation of the numerical model.

Comprehensive modeling of two-phase flows, where movement of two fluids is described by the same equations, and the location of the free surface is determined by interface tracking methods, usually require complicated and time-consuming calculations. Such models are also often characterized by poor numerical stability.

In the present paper authors propose a solution, based on the lattice Boltzmann method (LBM), allowing to take into account interactions between the movement of the liquid and gas phases, while maintaining simplicity of the numerical algorithm, characteristic for this method.

* M.Sc., ** Ph.D., *** Prof., Ph.D., D.Sc.: Faculty of Foundry Engineering, AGH University of Science and Technology, Kraków, Poland; e-mail: mszucki@agh.edu.pl 


\section{LATTICE BOLTZMANN METHOD}

\subsection{Single phase flows}

In the case of single-phase flows evolution of the model for LBM method is described by the lattice Boltzmann equation (LBE), which is generally formulated in the form of so-called approximation BGK (Bhatnagar-Gross-Krook):

$$
f_{i}\left(x+e_{i}, t+\Delta t\right)-f_{i}(x, t)=-\frac{1}{\tau}\left[f_{i}(x, t)-f_{i}^{e q}(x, t)\right],
$$

where, for considered here D2Q9 model (2 - dimensions, 9 - velocities): $i \in\{0,1, \ldots, 8\}$, $f$ - fluid particle distribution function, $\tau$ - relaxation time for the model determined by the kinematic visco-sity of the fluid.

This equation is solved on a regular grid with square cells (Fig. 1).

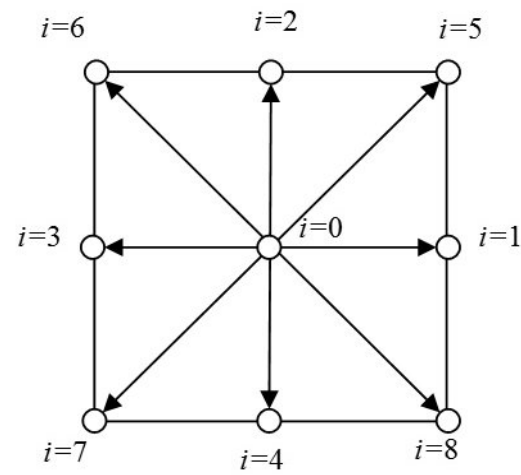

Fig. 1. Discrete velocity set for mesh cell

Equilibrium particle distribution function $f^{e q}$ is given by:

$$
f_{i}^{e q}(\rho(x, t), u(x, t))=f_{i}^{e q}(x, t)=\omega_{i} \rho\left[1+3\left(e_{i} \cdot u\right)+\frac{9}{2}\left(e_{i} \cdot u\right)^{2}-\frac{3}{2} u^{2}\right]
$$

where: $e_{i}$ is a set of dimensionless, discrete velocities. The values of other parameters from equation (2) are summarized in Table 1 [1].

Table 1. Parameters for D2Q9 model

\begin{tabular}{|c|c|c|}
\hline Parameters & $\omega$ & $0.5 e_{i}{ }^{2}$ \\
\hline$i=0$ & $4 / 9$ & 0 \\
\hline$i=1,2,3,4$ & $1 / 9$ & $1 / 2$ \\
\hline$i=5,6,7,8$ & $1 / 36$ & 1 \\
\hline
\end{tabular}


Local fluid properties such as density $\rho$ and velocity $u$ can be computed with

$$
\rho=\sum_{i=0}^{8} f_{i}, \quad \rho u=\sum_{i=0}^{8} f_{i} e_{i} .
$$

Equation (1) is usually solved in two steps Streaming and Collision:

Streaming:

$$
f_{i}(x, t)=f_{i}\left(x+e_{i n v}, t-\Delta t\right)
$$

Collision:

$$
f_{i}(x, t+\Delta t)=f_{i}(x, t)-\frac{1}{\tau}\left[f_{i}(x, t)-f_{i}^{e q}(x, t)\right]
$$

where inv denote the direction inverse to $i$.

\subsection{Free Surface Flows}

The present study is based on a free surface flow model for lattice Boltzmann method originally proposed in [2]. Some modifications to this method, also as examination of the possibility of its use to simulate the process of mould filling, were shown in study [3].

Application of this model involves the assignment of each grid cell to one of the three categories: cells completely filled by liquid (F), empty cells (E) and partially filled cells - so called interface cells (I). Movement of the liquid in cells I and F is described by the same equations (2-5) as in the case of single-phase flow. In turn, at the stage of computation the E cells are ignored. In this case, free surface tracking relies on calculation of mass transfer $m$ in the domain, and afterward determination of the local fraction of the liquid phase $\varepsilon$ in the cells I:

$$
\varepsilon=\frac{m}{\rho}
$$

Interface cell, which in, after last time step $\varepsilon \leq 0$ is converted to cell E, while I cell, where $\varepsilon \geq 1$ is transformed into cell F. After each step, the interface should be closed again, so there is no direct contact between fluids and empty cells. Therefore, the interface in this case represents a continuous boundary between the fluid and the gas phase.

In spite of its many advantages such as high performance and high accuracy, presented model of free surface flows, does not allow to simulate the motion of the gas phase (as mentioned empty cells are ignored). It only enables to determine the location of the interface, based on the average pressure exerted by gas on the liquid free surface. 


\section{PISTON MODEL}

In order to develop a simple and effective method for determination of pressure and velocity field in a area filled with gas, the authors proposed here a solution called the „piston model".

Omitting phenomena such as oxidation of the free surface or gas diffusion into the liquid, the interaction between the liquid metal and the gases that fills the mould can be reduced to a simple situation where alloy in the mould, like a piston in the cylinder, „pushes" and compresses gases, which in turn, are opposed to this movement through their increasing pressure $p_{g}$ (Fig. 2).

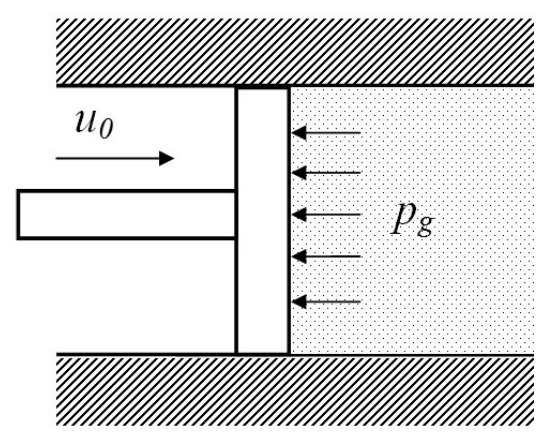

Fig. 2. Graphical interpretation of the proposed model

\subsection{Effect of movement of th free surface on velocity and pressure field in the gas phase}

For lattice Boltzmann method boundary condition that allows to transfer momentum of the moving free surface (piston) to the fluid can be put in the following form [4]:

$$
\begin{aligned}
f_{i}(x, t+\Delta t)= & w_{p}\left(f_{i n v}(x, t)+2 \omega_{i} \rho_{g} 3 e_{i} \cdot u_{0}\right)+\left(1-w_{p}\right) \\
& \left(f_{r}(x, t)+w_{r}(x, t) 2 \omega_{i} \rho_{g} 3 e_{i} \cdot\left[\left(n_{0} \cdot u_{0}\right) n_{o}\right]\right),
\end{aligned}
$$

where:

$f_{r}$ - distribution function resulting from the application of Free-slip boundary condition,

$\rho_{g}-$ fluid density,

$u_{0}$ - local velocity in interface cells,

$w_{r}$ - indicator which is equal to 1 when during streaming step function $f_{r}$ is reduced to No-slip reflection and 0 otherwise,

$w_{p}$ - indicator which is equal to 0 and 1 respectively for Free-slip and No-slip boundary condition on a free surface. 
Movement of gases (in the cells of type E) caused by equation (7), is treated in the numerical algorithm, as an ordinary single-phase flow. The presented approach, in terms of kinetics of movement in the gas phase, also denotes that with the parameter $w_{p}$ it is possible to define any boundary condition (Free-slip, Frictional-slip, No-slip [5]) on the metal free surface. It is noteworthy that for presented model it is possible to calculate local normal direction $n_{0}$ to the free surface directly, based on a fraction of liquid $\varepsilon$ in neighboring cells:

$$
n_{0}=0.5\left(\begin{array}{l}
\varepsilon_{j-1, k}-\varepsilon_{j+1, k} \\
\varepsilon_{j, k-1}-\varepsilon_{j, k+1}
\end{array}\right) \text {, }
$$

where: $j, k$ - Cartesian coordinates ( $x$ and $y$ respectively).

\subsection{Effect of gas pressure on the shape and movement of free surface}

A complete model of two-phase flows, should take into account not only the influence of movement of free surface on velocity and pressure field in the gas phase, but also effects of gas back-pressure on the motion of liquid metal. For the LBM free surface model described above, this task is quite simple because, as mentioned, numerical algorithm reflects the influence of average gas pressure on a location of the interface line (interfacial boundary).

In this case modifications will be reduced to replace the global gas pressure in the system, with local pressure calculated for each E cell adjacent to the interface. For the lattice Boltzmann method fluid pressure $p$ is closely related to its density $\rho$ according to the formula (for D2Q9 model):

$$
p=\frac{\rho}{3}
$$

Model presented above should ensure full two-way coupling between liquid and gas.

\section{MODEL VALIDATION}

To validate the proposed solution, a test domain (elbow), similar to that presented in [6] was chosen. In order to obtain the maximum stability of the numerical model, some modifications of object dimensions (Fig. 3) were made (leaving unchanged the relative proportions of the various components).

At the beginning of the process analyzed area is filled with gas and some portion of liquid, separated from each other by the interface line (free surface). After starting the calculation liquid flows into the domain on the left, compressing and ,pushing” the gas, which in turn flows out on the right side. In the numerical model the authors used physical 
parameters of water (liquid phase) and air (gas phase), took into account the gravity force $g=9.81 \mathrm{~m} / \mathrm{s}^{2}$ and assumed that the inflow velocity is $0.1 \mathrm{~m} / \mathrm{s}$ and at the outlet pressure is equal to atmospheric pressure $\rho_{\text {atm }}$ (fluid can freely enter the outlet). Tests were performed using two opposite boundary conditions on the domain walls: Free-slip (liquid freely "slides” at the boundary) and No-Slip (velocities tangential to the boundary are completely decelerated).

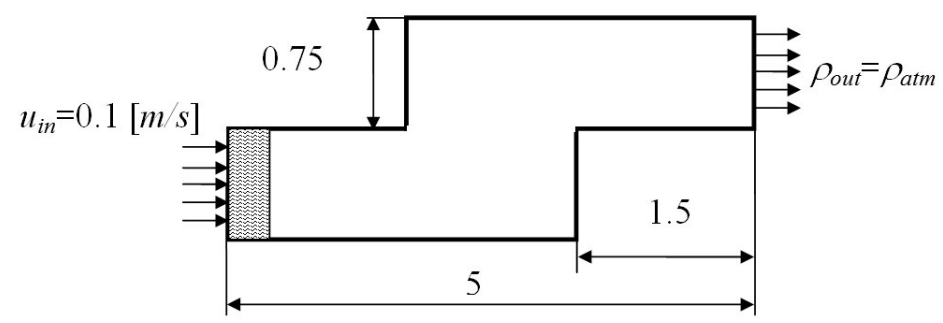

Fig. 3. Schematic introduction of the computational domain

Results from the presented model were compared with data (assumed as reference) from the commercial simulation software FLOW-3D. Both the velocity field in the gas phase as well as location of the free surface line were analyzed.

\section{ANALYSIS OF RESULTS AND SUMMARY}

For the No-slip boundary conditions (Fig. 4), the velocity fields obtained for the gas phase are very similar to results received during simulation in FLOW-3D. It may reflect the fact that the proposed model, with all adopted assumptions, allows for proper representation of the nature and kinetics of gas motion in the mould. For the Free-slip boundary condition (Fig. 5) the differences between the results derived from the proposed model and the data from commercial software are slightly higher, probably due to the simplifications assumed during the implementation of this condition. Also noteworthy is the fact that the shapes and location of area occupied by the liquid, obtained by modeling with the lattice Boltzmann method, were almost identical to those received in the FLOW-3D environment.

In each case, the greatest differences between obtained results and the reference data occur at the initial stage of simulations, and disappear with time. The correct interpretation of this phenomenon requires a further detailed analysis.

\section{CONCLUSIONS}

The proposed extended free surface model (piston model) allows simplified modeling of flows in the liquid - gas systems. Results obtained and summarized in the paper are highly compatible with the known and proven commercial solutions. The presented method is simple to implement and could be an interesting alternative to standard models of two-phase flows used in casting simulation software. 
a)

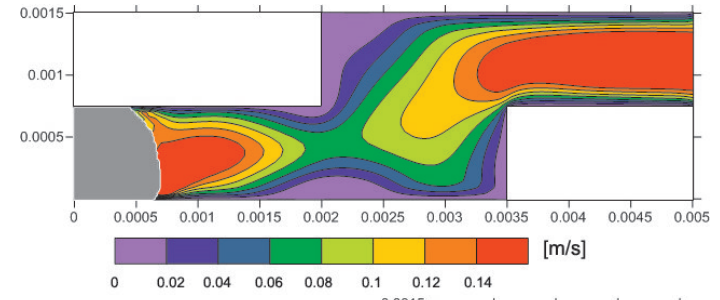

b)

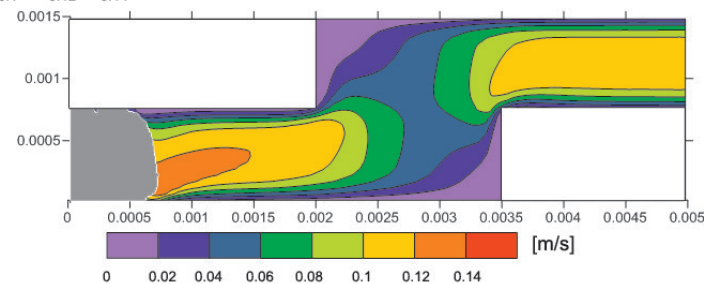

c)

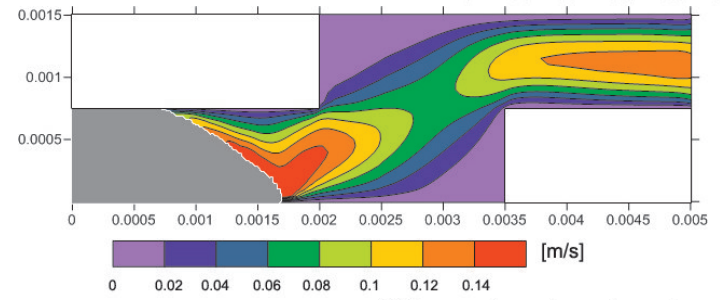

d)

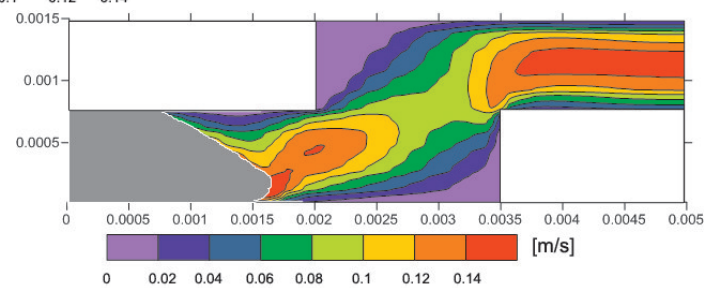

e)

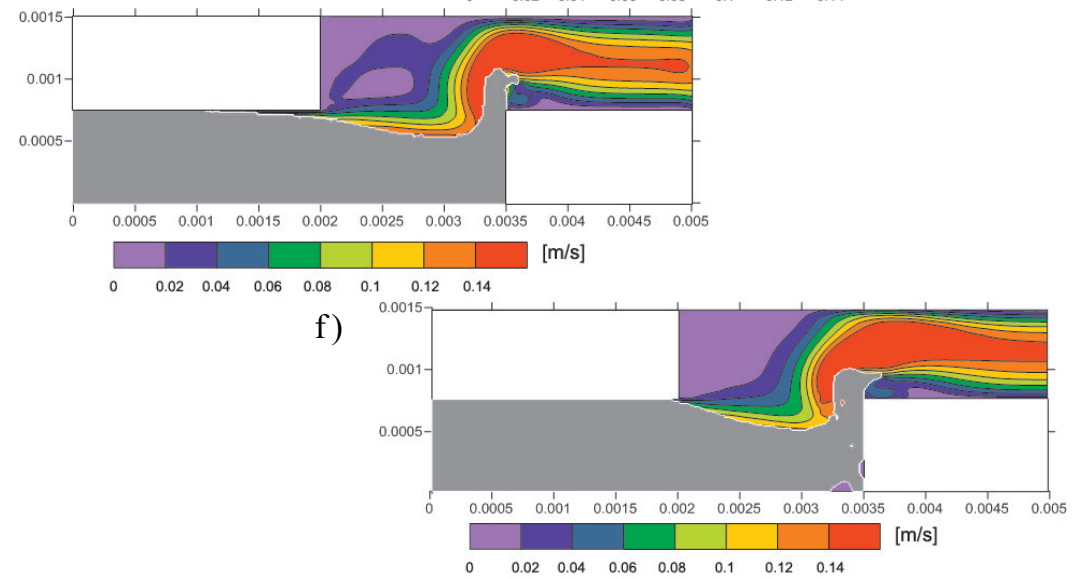

Fig. 4. Velocity field in the gas phase, in subsequent time steps for the proposed model (a, $c, e)$ and obtained by the simulation in FLOW-3D software $(b, d, f)$ for time: $t \approx 0.0038 \mathrm{~s}(a, b) ; t \approx 0.01 \mathrm{~s}$ $(c, d) ; t \approx 0.029 s(e, f)-$ No-slip boundary condition on the domain walls 
a)

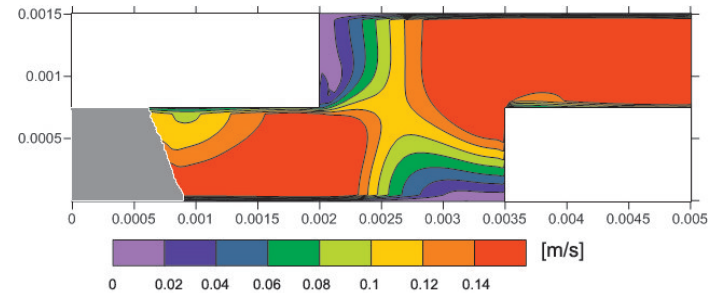

b)

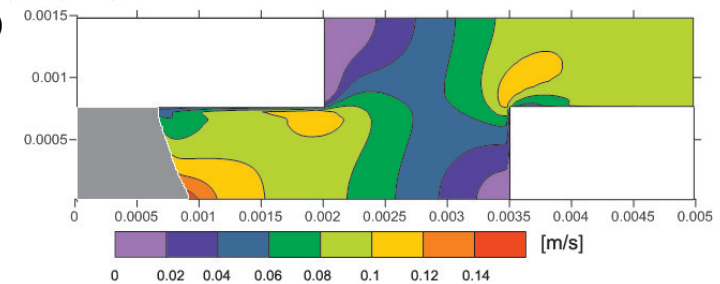

c)

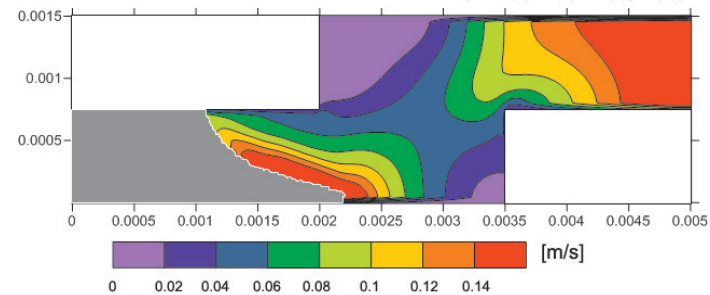

d)

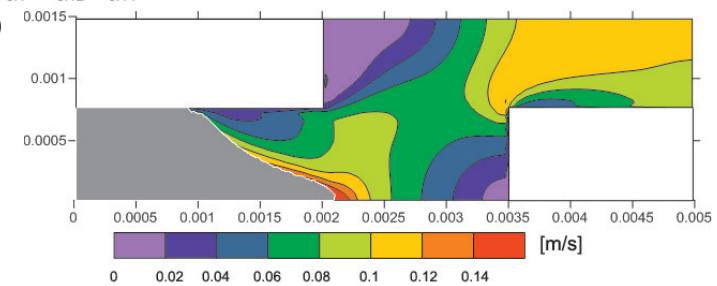

e)

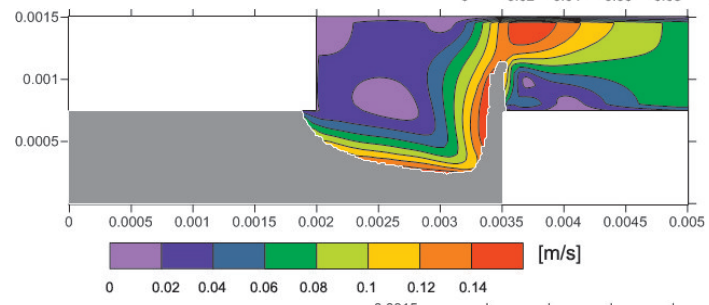

f)

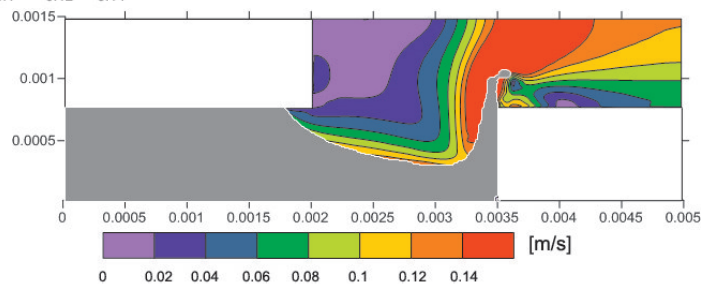

Fig. 5. Velocity field in the gas phase, in subsequent time steps for the proposed model (a, $c, e)$ and obtained by the simulation in FLOW-3D software $(b, d$, f) for time: $t \approx 0.005 s(a, b) ; t \approx 0.0124 \mathrm{~s}$ $(c, d) ; t \approx 0.025 s(e, f)$ - Free-slip boundary condition on the domain walls 


\section{Acknowledgements}

The authors acknowledge The European Community and The Polish Ministry of Science and Higher Education for financial support under Marie Curie Transfer of Knowledge grant No. MTKD-CT-2006-042468 (AGH No. 27.27.170.304) and grant No. N N508 480638 (AGH No. 18.18.170.383).

\section{REFERENCES}

[1] Koerner C.: Integral Foam Molding of Light Metals, Technology, Foam Physics and Foam Simulation, Springer, 2008

[2] Koerner C., Thies M., Hofmann T., Thurey N., Rude U.: Lattice Boltzmann Model for Free Surface Flow for Modeling Foaming, Journal of Stat. Phys., Vol. 121, 2005, 179-196

[3] Szucki M., Suchy J., Gurgul D.: Free surface reconstruction during modeling of mould filling process with Lattice Boltzmann Method, XXXIII International Conference Polish Foundry Day. Cracow 2009 (Conference proceedings)

[4] Thurey N.: Physically based Animation of Free Surface Flows with the Lattice Boltzmann Method, PhD Thesis, 2007

[5] Succi S.: The Lattice Boltzmann Equation for Fluid Dynamics and Beyond, Numerical Mathematics. and Scientific. Computation., Oxford University Press, 2001

[6] Cruchaga M., Celentano D., Tezduyar T.: Computation of mould filling processes with a moving Lagrangian interface technique, Communications. In Numerical. Methods in Engineering, Vol. 18, 2002, 483-493

Received

December 2010 